\title{
Editorial
}

\section{Laser Surgery in ENT - Head and Neck Surgery in Bangladesh}

Laser technology in the recent years has been introduced itself as an integral part of the therapeutic measures in many centers of otolaryngology, head and neck surgery. In this technique source is used on the basis of their characteristic wavelengths and the individual biophysical properties.

In the early 1970s, Jako coupled the Carbondioxide laser to the operating microscope and, thus, introduced this device into laryngology. Vaporization of laryngeal papillomas was one of the earliest indications. Though holding much promise this technique failed to be a solution for laryngeal papillomatosis. Similarly, the therapeutic benefits of the technique in the treatment of laryngeal and tracheal stenosis were over estimated, and in the treatment of the carcinoma of the larynx, oral cavity, and pharynx, the capability of the laser was for a long time underrated. Though Strong and Jako as early as in 1972 resected small vocal cord carcinoma restricted to the middle third of the vocal cord, laser surgery remained limited to small tumors of this kind.

In Europe, mainly in Germany, The $\mathrm{CO}_{2}$ laser came into use in the treatment of advanced laryngeal carcinoma in the late 1970s. This work was pioneered by Steiner, who had dissected and removed large tumors.

At the beginning of use of laser in ENT Surgery is not as simple as thought because important thing is to take decision regarding which type of laser to be used to treat a particular disease and which power density to apply.

Thus, the inexperienced surgeon would be well advised to attend one of the practical courses that offer an optimal introduction into laser surgery and should have some theoretical knowledge regarding the laser sources as well as the disease processes and its pathogenesis.

The most important application of lasers, particularly the $\mathrm{CO}_{2}$ laser, is in the treatment of upper aerodigestive tract carcinoma, especially if located in the larynx or hypopharynx. This method of treatment has been highly controversial in the past. However, due to the continual improvements in this therapy and its resulting successes the number of critics has dwindled over the years. In fact, this new approach has become increasingly accepted and implemented worldwide which is evidenced by courses and workshops which take place regularly at both the national and international levels.

Maiman was the first to describe laser in 1960. One year later, preliminary trials of the ruby laser, testing the feasibility of laser systems in an ophthalmologic application, were described by Campbell and Koester. The Argon laser and the $\mathrm{CO}_{2}$ laser were the first clinically applied laser systems in otorhinolaryngology. Meanwhile, various other diseases have been treated with a number of other laser systems, including the diode laser, ruby laser, HoYAG laser, ErYAG laser, NdYAG laser, yellow light laser as well as the dye laser for photodynamic therapy. Currently, the $\mathrm{CO}_{2}$ laser is the most widely used laser in otorhinolaryngology. Its main application is in the treatment of upper aerodigestive tract carcinoma. Other established indications of the $\mathrm{CO}_{2}$ laser include hypertrophic nasal conchae, reduction of hyperplastic tonsils of the base of the tongue, excision of laryngeal papillomas, and laser surgical myotomy of hypopharyngeal diverticula. Compared to the $\mathrm{CO}_{2}$ laser, the application range of the other laser systems is limited due to the specific biophysical characteristics of each type of laser, such as limited cutting quality or very selective (though useful) absorption of the laser light by pigments, especially hemoglobin. The later is significant in the treatment of hemangiomas and vascular malformation with the NdYAG laser. In the treatment of benign and malignant diseases of the upper aerodigestive tract, endoscopic and microscopic laser surgery is gaining everincreasing importance and widespread acceptance. This can be seen in the continuous increase in the number of congresses, courses, and publications on various aspects of laser surgery.

Jako and Strong, working in Boston, must be given credit for first introducing the $\mathrm{CO}_{2}$ laser in laryngeal 
microsurgery in the early 1970s. In the 1980s, the laser became more established in the treatment of benign lesions of the larynx, i.e. mainly in recurrent laryngeal papillomastosis. This is reflected by the extensive literature on the subject. On the other hand, laser was only slowly introduced into the treatment of malignancies and this development remained limited to only a few centers throughout the world. Furthermore, the application of laser largely remained limited to the excision of early vocal cord tumors. The first reports on successful outcomes of laser resection of vocal cord carcinomas were published by Strong and colleagues in 1975 . The limited number of indications precisely defined by these authors for laser tumor surgery was subsequently followed almost religiously, with only a few minor modifications being made. Burian and Hofler were the first in Europe to successfully treat a glottic carcinoma with a laser. Early in the 1980s, Steiner, working at that time at the Department of Otorhinolaryngology of the University of Erlangen, Germany, expanded the indication range of curative laser treatment to all regions and all tumor categories $(\mathrm{T} 1-\mathrm{T} 4)$.

The resection of laryngeal and hypopharyngeal carcinomas remains the main indication of transoral laser surgery. With the laser the surgeon is able to preserve the larynx and hypopharynx in many cases that previously called for laryngectomy as the sole surgical option. Moreover, benign lesions of the supraglottic larynx and hypopharynx, such as cyst, larygoceles and hypopharyngoceles, which used to require surgery by an external approach, have become amenable to being removed by transoral laser techniques.
In Bangladesh, introduction of laser in treating ENT diseases has changed the scenario, especially in treating recurrent laryngeal, pharyngeal carcinoma, different benign diseases of the larynx and pharynx like recurrent laryngeal respiratory papillomatosis ,treatment of allergic rhinitis like laser turbinoplasty and uvulopalatopharyngoplasty and excision of small tumors of the oral cavity and tongue. Although laser surgery takes more time for complete healing of operative wounds than conventional procedures, and costlier than the later, it is gaining rapid popularity amongst the surgeons and the patients because of its meticulous and precise nature as well as function preserving characteristic. More over conventional surgical techniques still remains as the last method after failure of the laser technique.

The main draw back of the procedure is its cost, availability of proper training facilities of the learners as well as the availability of the proper laser machines. All the problems can be solved if we can work together with heart and soul. We must mention that LASER is NOT the method of Tonsillectomy only as there is many simple ways to perform it.

So today laser surgery in ENT and HNS has definitely occupied a position where the aim is to preserve the organ. The trans-oral access introduces a new dimension to laser microsurgery: Precision Surgery as the surgery of the Future.

Md. Zillur Rahman, FCPS, MS

Senior Consultant, ENT

Apollo Hospitals, Dhaka, Bangladesh 